\title{
Mixing of fermions and spectral representation of propagator
}

\section{A.E. Kaloshin*}

Irkutsk State University, Irkutsk, Russia

E-mail: kaloshin@physdep.isu.ru

\section{V.P. Lomov}

Institute for System Dynamics and Control Theory, RAS, Irkutsk, Russia

E-mail: lomov.vi@icc.ru

We develop the spectral representation of propagator for $n$ mixing fermion fields in case of Pparity violation. Solving of the eigenstate problem for inverse matrix propagator allows to build the system of orthogonal projectors and to represent the matrix propagator as a sum of poles with positive and negative energy. The procedure of multiplicative renormalization is investigated, the renormalization matrices are obtained in a closed form without using of pertubation theory.

XXII International Baldin Seminar on High Energy Physics Problems,

15-20 September 2014

JINR, Dubna, Russia

${ }^{*}$ Speaker. 


\section{Introduction}

Problem of neutrino oscillations is in the center of attention last decades, both from experimental and theoretical points of view. This phenomenon is generated by mixing in system of neutrinos, when mass states differ from flavor ones. Since quantum field theory is a proper theoretical base for description of these effects, the essential efforts were devoted to application of QFT methods for mixing in system of neutrinos $[1,2,3,4,5,6,7]$. We mentioned here only small part of relevant publications (see also the references cited therein), directly related with problem of neutrino oscillations. Mixing effects play also an essential role in system of quarks, where QFT effects lead to renormalization of Cabbibo-Kobayashi-Maskawa (CKM) matrix and its evolution with energy (see, e.g. $[8,9,10,11]$ ).

In recent series of papers $[12,13,14]$ the properties of matrix dressed propagator in presence of P-parity violation were investigated in detail. The dressed propagator was represented in a closed algebraic form, which satisfies the main physical requirements and allows to build the renormalized propagator. The pole scheme of renormalization was investigated and wave-function renormalization (WFR) matrices were obtained in a closed analytical form without recourse to perturbation theory.

Here we present some special algebraic construction for consideration of fermion mixing in QFT frameworks. The main feature of suggested construction is that propagator is represented as a sum of single poles with positive and negative energy. Note, that it is made in a covariant manner $1 /\left(W \pm m_{i}\right)$ and this property follows from use of the off-shell projection operators $\Lambda^{ \pm}(2.1)$ at first step. The obtained very simple expression for WFR matrices (4.21) confirms the old opinion that just $W$ is the natural variable in fermion case.

Technically, the suggested construction is based on so called spectral representation of operator (see, e.g. textbook [15]). In this representation the self-adjoint operator $\hat{A}$ takes the form (in quantum-mechanical notations):

$$
\hat{A}=\sum_{i} \lambda_{i}|i\rangle\langle i|=\sum_{i} \lambda_{i} \Pi_{i}
$$

where $\lambda_{i}$ are eigenvalues of operator, $|i\rangle$ are eigenvectors

$$
\hat{A}|i\rangle=\lambda_{i}|i\rangle,
$$

and $\Pi_{i}=|i\rangle\langle i|$ are corresponding orthogonal projectors (eigenprojectors). In case of non-selfadjoint operator the similar decomposition also exists but to construct it, one needs solutions of both left and right eigenstate problems.

If we have $n$ fermion fields with the same quantum numbers, they begin to mix at loop level even in the case of diagonal mass matrix. In QFT the main object of studying is the dressed matrix propagator $G(p)$. To build the spectral representation of $G(p)$, first of all one needs to solve the eigenstate problem for inverse propagator $S(p)^{1}$

$$
S \Pi_{i}=\lambda_{i} \Pi_{i} .
$$

\footnotetext{
${ }^{1}$ Here $S$ (and $\Pi$ also) has two sets of indexes $S_{\alpha \beta ; i j}$, where $\alpha, \beta=1, \ldots, 4$ are the Dirac $\gamma$-matrix indexes and $i, j=$ $1, \ldots, n$ are generation indexes. Note that, following to [16], we are looking for eigenprojectors instead of eigenvectors to avoid cumbersome intermediate expressions.
} 
If we have the complete system of orthogonal eigenprojectors

$$
\Pi_{i} \Pi_{k}=\delta_{i k} \Pi_{k},
$$

then we come to spectral representation of inverse propagator $S(p)$

$$
S(p)=\sum_{i=1}^{2 n} \lambda_{i} \Pi_{i}
$$

After it the matrix propagator $G(p)$ is obtained by reversing of (1.3)

$$
G(p)=\sum_{i=1}^{2 n} \frac{1}{\lambda_{i}} \Pi_{i}
$$

If projectors possess the orthogonality property, then the same $\Pi_{i}$ is solution of two eigenstate problems: left (1.1) and right one

$$
\Pi_{i} S=\lambda_{i} \Pi_{i}
$$

As it will seen below, the representation (1.3) looks very simple and evident in case of Pparity conservation, so the main technical problems are related with appearance of $\gamma^{5}$ in vertex and dressed propagator. In [16] we constructed the representation (1.3) for single fermion $(n=1)$ in case of parity violation and investigated the renormalization procedure. Here we consider the case of $n$ mixing fermion fields, construct the eigenprojectors $\Pi_{i}$, investigate the main algebraic properties and procedure of multiplicative renormalization.

\section{Eigenstate problem for matrix inverse propagator}

\subsection{Preliminaries}

In the following it's convenient to use the off-shell $\gamma$-matrix projectors ${ }^{2}$

$$
\Lambda^{ \pm}(p)=\frac{1}{2}\left(1 \pm \frac{\hat{p}}{W}\right)
$$

where $W=\sqrt{p^{2}}$ is the center mass energy.

In case of parity conservation the eigenprojectors $\Pi_{i}$ are just $\Lambda^{ \pm}$, multiplied by flavor matrix, see (2.8) below. In theory with $\gamma^{5}$ the $\gamma$-matrix projectors $\Lambda^{ \pm}$appear at intermediate stage of the $\Pi_{i}$ building but they are useful to simplify the algebra.

In case of parity violation we introduce the following set of matrices

$$
\mathscr{P}_{1}=\Lambda^{+}, \quad \mathscr{P}_{2}=\Lambda^{-}, \quad \mathscr{P}_{3}=\Lambda^{+} \gamma^{5}, \quad \mathscr{P}_{4}=\Lambda^{-} \gamma^{5}
$$

and use them as a basis for self-energy and propagator.

\footnotetext{
${ }^{2}$ Many people used these off-shell projectors for different purposes, the first known for us case is related with problem of fermion Regge poles, see papers of V.N. Gribov and co-authors [17, 18]. Thanks to N.N. Achasov for indication of these references.
} 
Then the matrix inverse propagator may be written as a decomposition

$$
S(p)=G^{-1}(p)=\sum_{M=1}^{4} \mathscr{P}_{M} S_{M}(W)
$$

where the matrix coefficients $S_{M}$ have the obvious symmetry properties:

$$
S_{2}(W)=S_{1}(-W), \quad S_{4}(W)=S_{3}(-W)
$$

and are calculated as

$$
\begin{aligned}
S_{1} & =\frac{1}{2} \operatorname{Sp}\left(\mathscr{P}_{1} S\right), & S_{2} & =\frac{1}{2} \operatorname{Sp}\left(\mathscr{P}_{2} S\right) \\
S_{3} & =\frac{1}{2} \operatorname{Sp}\left(\mathscr{P}_{4} S\right), & S_{4} & =\frac{1}{2} \operatorname{Sp}\left(\mathscr{P}_{3} S\right) .
\end{aligned}
$$

- If parity is conserved, the self-energy

$$
\Sigma(p) \equiv A\left(p^{2}\right)+\hat{p} B\left(p^{2}\right)=\mathscr{P}_{1}\left(A\left(W^{2}\right)+W B\left(W^{2}\right)\right)+\mathscr{P}_{2}\left(A\left(W^{2}\right)-W B\left(W^{2}\right)\right)
$$

contains only two terms in the decomposition (2.3). In this case the eigenstate problem (1.1) is reduced to eigenstate problem for $n \times n$ matrices $S_{1,2}$.

$$
\begin{aligned}
& S_{1} \pi_{1} \equiv\left(A\left(W^{2}\right)+W B\left(W^{2}\right)\right) \pi_{1}=\lambda \pi_{1}, \\
& S_{2} \pi_{2} \equiv\left(A\left(W^{2}\right)-W B\left(W^{2}\right)\right) \pi_{2}=\lambda \pi_{2}
\end{aligned}
$$

and eigenprojectors $\Pi_{i}$ take the factorized form

$$
\begin{array}{ll}
\Pi_{i}=\Lambda^{+} \pi_{1}^{(i)}, & i=1, \ldots, n \\
\Pi_{i}=\Lambda^{-} \pi_{2}^{(i)}, & i=n+1, \ldots, 2 n
\end{array}
$$

for positive and negative energy poles correspondingly.

- If P-parity is violated, the spectral representation (1.3) for inverse propagator becomes less evident. For single fermion ( $n=1$ in above) it was built and investigated in [16]. The eigenvalues $\lambda_{1,2}(W)$ are defined by characteristic equation

$$
\lambda^{2}-\lambda\left(S_{1}+S_{2}\right)+\left(S_{1} S_{2}-S_{3} S_{4}\right)=0,
$$

where the numbers $S_{i}$ are coefficients in decomposition (2.3). The eigenprojectors in general case are

$$
\begin{aligned}
& \Pi_{1}=\frac{1}{\lambda_{2}-\lambda_{1}}\left(\left(S_{2}-\lambda_{1}\right) \mathscr{P}_{1}+\left(S_{1}-\lambda_{1}\right) \mathscr{P}_{2}-S_{3} \mathscr{P}_{3}-S_{4} \mathscr{P}_{4}\right), \\
& \Pi_{2}=\frac{1}{\lambda_{1}-\lambda_{2}}\left(\left(S_{2}-\lambda_{2}\right) \mathscr{P}_{1}+\left(S_{1}-\lambda_{2}\right) \mathscr{P}_{2}-S_{3} \mathscr{P}_{3}-S_{4} \mathscr{P}_{4}\right) .
\end{aligned}
$$

If to return in the final answer to $\gamma$-matrix components

$$
S=a+\hat{n} b+\gamma^{5} c+\hat{n} \gamma^{5} d=a+\hat{n}\left(b+\hat{n} \gamma^{5} c+\gamma^{5} d\right),
$$

where $n^{\mu}=p^{\mu} / W$ is the unit vector, then the eigenprojectors may be re-written in the very simple form

$$
\Pi_{1,2}=\frac{1}{2}\left(1 \pm \hat{n} \cdot \frac{b+\hat{n} \gamma^{5} c+\gamma^{5} d}{\sqrt{b^{2}+c^{2}-d^{2}}}\right)
$$




\subsection{Left eigenstate problem}

Let us consider the mixing problem with $n$ fermion fields and parity violation. The inverse propagator is defined by decomposition (2.3) with arbitrary matrix coefficients $S_{M}(W)$. Following [16], we solve the eigenstate problem

$$
S \Pi=\lambda \Pi
$$

in matrix form, i.e. from the beginning we are looking for eigenprojectors $\Pi$ instead of eigenvectors. The looked for eigenprojectors also may be written as decomposition (2.3)

$$
\Pi=\sum_{M=1}^{4} \mathscr{P}_{M} A_{M},
$$

with matrix $n \times n$ coefficients $A_{M}(W)$. Due to simple multiplicative properties of the basis (2.2), it's easy to reduce the eigenstate problem (2.13) to the following set of linear equations for unknown matrices $A_{M}$

$$
\begin{aligned}
& \left(S_{1}-\lambda\right) A_{1}+S_{3} A_{4}=0 \\
& \left(S_{2}-\lambda\right) A_{2}+S_{4} A_{3}=0 \\
& \left(S_{1}-\lambda\right) A_{3}+S_{3} A_{2}=0 \\
& \left(S_{2}-\lambda\right) A_{4}+S_{4} A_{1}=0
\end{aligned}
$$

In fact we have two separated subsystems for unknown $A_{1}, A_{4}$ and $A_{2}, A_{3}$, so it's convenient to express $A_{3}, A_{4}$ by

$$
A_{3}=-S_{4}^{-1}\left(S_{2}-\lambda\right) A_{2}, \quad A_{4}=-S_{3}^{-1}\left(S_{1}-\lambda\right) A_{1}
$$

and to obtain the homogeneous equations for $n \times n$ matrices $A_{1}, A_{2}$

$$
\begin{aligned}
\hat{O} A_{1} & \equiv\left[\left(S_{2}-\lambda\right) S_{3}^{-1}\left(S_{1}-\lambda\right)-S_{4}\right] A_{1}=0, \\
\hat{O}^{\prime} A_{2} & \equiv\left[\left(S_{1}-\lambda\right) S_{4}^{-1}\left(S_{2}-\lambda\right)-S_{3}\right] A_{2}=0 .
\end{aligned}
$$

Here we introduced the short notations $\hat{O}, \hat{O}^{\prime}$ for emerged $\lambda$-dependent operators. One can see that matrices $\hat{O}, \hat{O}^{\prime}$ are related with each other by similarity relations

$$
\hat{O}^{\prime}=\left(S_{1}-\lambda\right) S_{4}^{-1} \cdot \hat{O} \cdot\left(S_{1}-\lambda\right)^{-1} S_{3}=S_{3}\left(S_{2}-\lambda\right)^{-1} \cdot \hat{O} \cdot S_{4}^{-1}\left(S_{2}-\lambda\right),
$$

so equations (2.17) give the same characteristic equation for $\lambda$

$$
\operatorname{det}\left[\left(S_{2}-\lambda\right) S_{3}^{-1}\left(S_{1}-\lambda\right)-S_{4}\right]=0 .
$$

In the absence of degeneration this equation gives $2 n$ different eigenvalues $\lambda_{i}(W)$.

Thus the matrix solution of left eigenstate problem (2.13) may be written as

$$
\Pi^{i}=\mathscr{P}_{1} A_{1}^{i}+\mathscr{P}_{2} A_{2}^{i}-\mathscr{P}_{3} S_{4}^{-1}\left(S_{2}-\lambda_{i}\right) A_{2}^{i}-\mathscr{P}_{4} S_{3}^{-1}\left(S_{1}-\lambda_{i}\right) A_{1}^{i},
$$

where $A_{1}^{i}, A_{2}^{i}$ are solutions of equations

$$
\begin{aligned}
& \hat{O}_{i} A_{1}^{i} \equiv \hat{O}\left(\lambda=\lambda_{i}\right) A_{1}^{i}=0, \\
& \hat{O}_{i}^{\prime} A_{2}^{i} \equiv \hat{O}^{\prime}\left(\lambda=\lambda_{i}\right) A_{2}^{i}=0
\end{aligned}
$$

and eigenvalues $\lambda_{i}(W)$ are defined by equation (2.19). 


\subsection{Right eigenstate problem}

It was noted in above that orthogonal projectors should satisfy both left and right eigenstate problems. So as the next step consider the right eigenstate problem for inverse propagator

$$
\Pi_{R} S=\lambda \Pi_{R}
$$

We can look for the right eigenprojectors $\Pi_{R}$ in the same form (2.14) with matrix coefficients $B_{M}$. Similar calculations give the matrix solution of the right problem

$$
\Pi_{R}^{i}=\mathscr{P}_{1} B_{1}^{i}+\mathscr{P}_{2} B_{2}^{i}-\mathscr{P}_{3} B_{1}^{i} S_{3}\left(S_{2}-\lambda_{i}\right)^{-1}-\mathscr{P}_{4} B_{2}^{i} S_{4}\left(S_{1}-\lambda_{i}\right)^{-1},
$$

where $B_{1}^{i}, B_{2}^{i}$ are solutions of the left homogeneous equations

$$
B_{1}^{i} \hat{O}_{i}^{\prime}=0, \quad B_{2}^{i} \hat{O}_{i}=0
$$

and eigenvalues $\lambda_{i}(W)$ are defined by the same equation (2.19).

\subsection{Left and right together}

Let us require the eigenmatrix $\Pi$ to be solution of both left and right eigenstate problems. It means that expressions (2.20), (2.23) should coincide with each other.

First of all $B_{1}^{i}=A_{1}^{i}, B_{2}^{i}=A_{2}^{i}$, as it seen from $\mathscr{P}_{1}, \mathscr{P}_{2}$ terms. Coefficients at $\mathscr{P}_{3}, \mathscr{P}_{4}$ give two relations between $A_{1}$ and $A_{2}$

$$
\begin{aligned}
& A_{2}^{i}=S_{3}^{-1}\left(S_{1}-\lambda_{i}\right) \cdot A_{1}^{i} \cdot S_{3}\left(S_{2}-\lambda_{i}\right)^{-1}, \\
& A_{2}^{i}=\left(S_{2}-\lambda_{i}\right)^{-1} S_{4} \cdot A_{1}^{i} \cdot\left(S_{1}-\lambda_{i}\right) S_{4}^{-1} .
\end{aligned}
$$

Now the matrices $A_{1}, A_{2}$ satisfy both left and right homogeneous equations

$$
\begin{array}{ll}
\hat{O}_{i} A_{1}^{i}=0, & A_{1}^{i} \hat{O}_{i}^{\prime}=0, \\
\hat{O}_{i}^{\prime} A_{2}^{i}=0, & A_{2}^{i} \hat{O}_{i}=0 .
\end{array}
$$

Note that homogeneous equations for $A_{1}$ lead to following equalities (only for solutions $A_{1}^{i}$ )

$$
\begin{aligned}
& S_{3}^{-1}\left(S_{1}-\lambda_{i}\right) \cdot A_{1}^{i}=\left(S_{2}-\lambda_{i}\right)^{-1} S_{4} \cdot A_{1}^{i}, \\
& A_{1}^{i} \cdot\left(S_{1}-\lambda_{i}\right) S_{4}^{-1}=A_{1}^{i} \cdot S_{3}\left(S_{2}-\lambda_{i}\right)^{-1},
\end{aligned}
$$

so one can see that two relations (2.25) actually coincide. Moreover, one can convince yourself that equations for $A_{2}^{i}$ (2.26) are consequence of relation (2.25) and equations for $A_{1}^{i}$. Therefore, it is sufficient to require the left and right homogeneous equations for $A_{1}^{i}$ and connection between $A_{2}^{i}$ and $A_{1}^{i}$.

At last, note that the matrix $A_{1}^{i}$ has zeroth determinant and may be represented in the splitted form

$$
A_{1}^{i}=\psi_{i}\left(\tilde{\psi}_{i}\right)^{\mathrm{T}},
$$

where vectors $\psi_{i}, \tilde{\psi}_{i}$ (columns) are solutions of homogeneous equations

$$
\hat{O}_{i} \psi_{i}=0, \quad\left(\tilde{\psi}_{i}\right)^{\mathrm{T}} \hat{O}_{i}^{\prime}=0 \quad\left(\text { or }\left(\hat{O}_{i}^{\prime}\right)^{\mathrm{T}} \tilde{\psi}_{i}=0\right) .
$$


Then solution of both left and right eigenstate problems may be represented as

$$
\begin{aligned}
\Pi_{i}=\mathscr{P}_{1} \psi_{i}\left(\tilde{\psi}_{i}\right)^{\mathrm{T}}+\mathscr{P}_{2} S_{3}^{-1}\left(S_{1}-\lambda_{i}\right) \psi_{i}\left(\tilde{\psi}_{i}\right)^{\mathrm{T}}\left(S_{1}-\lambda_{i}\right) S_{4}^{-1}- \\
\quad-\mathscr{P}_{3} \psi_{i}\left(\tilde{\psi}_{i}\right)^{\mathrm{T}}\left(S_{1}-\lambda_{i}\right) S_{4}^{-1}-\mathscr{P}_{4} S_{3}^{-1}\left(S_{1}-\lambda_{i}\right) \psi_{i}\left(\tilde{\psi}_{i}\right)^{\mathrm{T}} .
\end{aligned}
$$

For short notations it is convenient to introduce the vectors $\phi_{i}, \tilde{\phi}_{i}$ as

$$
\phi_{i}=S_{3}^{-1}\left(S_{1}-\lambda_{i}\right) \psi_{i}, \quad\left(\tilde{\phi}_{i}\right)^{\mathrm{T}}=\left(\tilde{\psi}_{i}\right)^{\mathrm{T}}\left(S_{1}-\lambda_{i}\right) S_{4}^{-1} .
$$

In these terms the "matrix" $\Pi_{i}$, which is a solution of both left and right eigenvalue problems, takes very elegant form

$$
\Pi_{i}=\mathscr{P}_{1} \cdot \psi_{i}\left(\tilde{\psi}_{i}\right)^{\mathrm{T}}+\mathscr{P}_{2} \cdot \phi_{i}\left(\tilde{\phi}_{i}\right)^{\mathrm{T}}-\mathscr{P}_{3} \cdot \psi_{i}\left(\tilde{\phi}_{i}\right)^{\mathrm{T}}-\mathscr{P}_{4} \cdot \phi_{i}\left(\tilde{\psi}_{i}\right)^{\mathrm{T}}
$$

Recall, that the auxiliary vectors $\phi_{i}, \tilde{\phi}_{i}$ satisfy the following homogeneous equations (consequence of definition)

$$
\hat{O}_{i}^{\prime} \phi_{i}=0, \quad\left(\tilde{\phi}_{i}\right)^{\mathrm{T}} \hat{O}_{i}=0 .
$$

\subsection{Eigenprojectors}

So we have $\Pi_{i}(2.32)$ - solution of both left and right eigenstate problems. Let us require these "matrices" (with two sets of indexes) $\Pi_{i}$ to be orthogonal projectors

$$
\Pi_{i} \Pi_{k}=\delta_{i k} \Pi_{k} .
$$

It gives four equations of the form

$$
\begin{aligned}
& \psi_{i}\left[\left(\tilde{\psi}_{i}\right)^{\mathrm{T}} \psi_{k}+\left(\tilde{\phi}_{i}\right)^{\mathrm{T}} \phi_{k}-\delta_{i k}\right]\left(\tilde{\psi}_{k}\right)^{\mathrm{T}}=0, \\
& \phi_{i}\left[\left(\tilde{\psi}_{i}\right)^{\mathrm{T}} \psi_{k}+\left(\tilde{\phi}_{i}\right)^{\mathrm{T}} \phi_{k}-\delta_{i k}\right]\left(\tilde{\psi}_{k}\right)^{\mathrm{T}}=0, \\
& \psi_{i}\left[\left(\tilde{\psi}_{i}\right)^{\mathrm{T}} \psi_{k}+\left(\tilde{\phi}_{i}\right)^{\mathrm{T}} \phi_{k}-\delta_{i k}\right]\left(\tilde{\phi}_{k}\right)^{\mathrm{T}}=0, \\
& \phi_{i}\left[\left(\tilde{\psi}_{i}\right)^{\mathrm{T}} \psi_{k}+\left(\tilde{\phi}_{i}\right)^{\mathrm{T}} \phi_{k}-\delta_{i k}\right]\left(\tilde{\phi}_{k}\right)^{\mathrm{T}}=0,
\end{aligned}
$$

which are equivalent to the orthonormality condition for vectors involved in (2.32)

$$
\left(\tilde{\psi}_{i}\right)^{\mathrm{T}} \psi_{k}+\left(\tilde{\phi}_{i}\right)^{\mathrm{T}} \phi_{k}=\delta_{i k}
$$

- If $i \neq k$ the condition (2.36) is consequence of equation on $\psi_{k}$ and $\left(\tilde{\psi}_{i}\right)^{\mathrm{T}}$. To see it, let us rewrite (2.36) in terms of the vectors $\psi_{i}$ and $\tilde{\phi}_{i}$ :

$$
\left(\tilde{\phi}_{i}\right)^{\mathrm{T}}\left[\left(S_{2}-\lambda_{i}\right) S_{3}^{-1}+S_{3}^{-1}\left(S_{1}-\lambda_{k}\right)\right] \psi_{k}=\delta_{i k} .
$$

Now let us write down the homogeneous equations for $\psi_{k}$ and $\tilde{\phi}_{i}$

$$
\begin{aligned}
& 0=\hat{O}_{k} \psi_{k}=\left[S_{3}^{-1} \lambda_{k}^{2}-\lambda_{k}\left(S_{2} S_{3}^{-1}+S_{3}^{-1} S_{1}\right)+S_{2} S_{3}^{-1} S_{1}-S_{4}\right] \psi_{k}, \\
& 0=\left(\tilde{\phi}_{i}\right)^{\mathrm{T}} \hat{O}_{i}=\left(\tilde{\phi}_{i}\right)^{\mathrm{T}}\left[S_{3}^{-1} \lambda_{i}^{2}-\lambda_{i}\left(S_{2} S_{3}^{-1}+S_{3}^{-1} S_{1}\right)+S_{2} S_{3}^{-1} S_{1}-S_{4}\right] .
\end{aligned}
$$


Multiplying first of these equations by $\left(\tilde{\phi}_{i}\right)^{\mathrm{T}}$ from the left, second one by $\psi_{k}$ from the right, and subtracting one equation from another, we obtain

$$
\left(\lambda_{k}-\lambda_{i}\right)\left(\tilde{\phi}_{i}\right)^{\mathrm{T}}\left[\left(S_{2}-\lambda_{i}\right) S_{3}^{-1}+S_{3}^{-1}\left(S_{1}-\lambda_{k}\right)\right] \psi_{k}=0,
$$

and at $\lambda_{i} \neq \lambda_{k}$ it gives the condition (2.36).

- At $i=k$ (2.36) defines the normalization (with weight) of the vector $\psi_{i}$ in respect to $\tilde{\psi}_{i}$.

\section{Case of $C P$ conservation}

In case of $\mathrm{CP}$ conservation the self-energy contributions

$$
\Sigma(p)=\sum_{M=1}^{4} \mathscr{P}_{M} \Sigma_{M}(W)=A\left(p^{2}\right)+\hat{p} B\left(p^{2}\right)+\gamma^{5} C\left(p^{2}\right)+\hat{p} \gamma^{5} D\left(p^{2}\right)
$$

have the following symmetry properties (see, e.g. [19])

$$
A^{\mathrm{T}}=A, \quad B^{\mathrm{T}}=B, \quad D^{\mathrm{T}}=D, \quad C^{\mathrm{T}}=-C,
$$

which are equivalent to

$$
\left(\Sigma_{1,2}\right)^{\mathrm{T}}=\Sigma_{1,2}, \quad\left(\Sigma_{3}\right)^{\mathrm{T}}=-\Sigma_{4}
$$

Since the inverse propagator $S(p)$ has the same symmetry properties (3.3) and it connects matrices $\hat{O}$ and $\hat{O}^{\prime}$

$$
\hat{O}^{\prime}=-(\hat{O})^{\mathrm{T}}
$$

Eigenprojectors have the form (2.32) but now two equations (2.29) coincide

$$
\hat{O}_{i} \psi_{i}=0, \quad \hat{O}_{i} \tilde{\psi}_{i}=0 .
$$

Then (in absence of degeneration) $\tilde{\psi}_{i}=c \psi_{i}$ and, redefining vectors $\sqrt{c} \psi_{i} \rightarrow \psi_{i}$, we obtain eigenprojectors in the form

$$
\Pi_{i}=\mathscr{P}_{1} \cdot \psi_{i}\left(\psi_{i}\right)^{\mathrm{T}}-\mathscr{P}_{2} \cdot \phi_{i}\left(\phi_{i}\right)^{\mathrm{T}}+\mathscr{P}_{3} \cdot \psi_{i}\left(\phi_{i}\right)^{\mathrm{T}}-\mathscr{P}_{4} \cdot \phi_{i}\left(\psi_{i}\right)^{\mathrm{T}}
$$

In case of CP conservation we need to solve only one homogeneous equation for $\psi_{i}$

$$
\hat{O}_{i} \psi_{i}=0
$$

vector $\phi_{i}$ is related with $\psi_{i}$ by

$$
\phi_{i}=S_{3}^{-1}\left(S_{1}-\lambda_{i}\right) \psi_{i}, \quad \text { or } \quad\left(\phi_{i}\right)^{\mathrm{T}}=-\left(\psi_{i}\right)^{\mathrm{T}}\left(S_{1}-\lambda_{i}\right) S_{4}^{-1}
$$

and satisfies the homogeneous equation (consequence of (3.7), (3.8))

$$
\hat{O}_{i}^{T} \phi_{i}=0 \text {. }
$$

The orthonormality condition $\Pi_{i} \Pi_{k}=\delta_{i k} \Pi_{k}$ leads to simple property of vectors

$$
\left(\psi_{i}\right)^{\mathrm{T}} \psi_{k}-\left(\phi_{i}\right)^{\mathrm{T}} \phi_{k}=\delta_{i k} .
$$

As it was shown before, this is not a new requirement: at $i \neq k$ it follows from homogeneous equation and at $i=k$ it defines normalization of vectors $\psi_{i}$. 


\section{Multiplicative renormalization}

We will consider the multiplicative renormalization (wave-function renormalization) of matrix propagator $G(p)$. This issue was investigated in detail in [20], so our main purpose here is to reformulate this scheme in terms of spectral representation.

If the renormalized dressed matrix propagator $G^{\text {ren }}(p)$ has poles at points $m_{l}$ we can put the eigenvalues $\lambda_{l}(W)$ in the same order, so that $\lambda_{l}\left(m_{l}\right)=0, l=1 \ldots 2 n$. In vicinity of point $\hat{p}=m_{l}$ matrix propagator has the form

$$
G^{\mathrm{ren}}(p) \sim\left(\begin{array}{c}
\vdots \\
\cdots \frac{1}{\hat{p}-m_{l}} \cdots \\
\vdots
\end{array}\right),
$$

where $\left(G^{\text {ren }}\right)_{l l}$ has pole with unit residue and other elements of $G^{\text {ren }}(p)$ are regular at $\hat{p} \rightarrow m_{l}$. It is convenient to renormalize the inverse matrix propagator $S(p)$, so we need to know its behaviour in vicinity of pole. It was investigated in [20], result may be presented in the form

$$
S_{i j}^{\text {ren }} \underset{\hat{p} \rightarrow m_{l}}{\longrightarrow} \begin{cases}\hat{p}-m_{l}, & i=l, j=l \\ M^{i l}\left(\hat{p}-m_{l}\right), & i \neq l, j=l \\ \left(\hat{p}-m_{l}\right) M^{l j}, & i=l, j \neq l \\ \text { arbitrary, } & i \neq l, j \neq l\end{cases}
$$

where matrices $M^{i l}, M^{l j}$ can be non-commutative with $\hat{p}-m_{l}$ because of $\gamma^{5}$. If to write down decomposition of $S^{\text {ren }}$ in our basis

$$
S^{\mathrm{ren}}(p)=\sum_{M=1}^{4} \mathscr{P}_{M} S_{M}^{\mathrm{ren}}(W)
$$

we can reformulate the requirements (4.2) in terms of this decomposition.

Note that the limit $\hat{p} \rightarrow m_{l}$ means that $p^{2} \rightarrow m_{l}^{2}$ or $W \rightarrow \pm m_{l}$. One can see that with use of decomposition (4.3), it's sufficient to investigate only $W \rightarrow m_{l}$ limit (positive energy pole in propagator) since the symmetry properties $S_{2}(W)=S_{1}(-W), S_{4}(W)=S_{3}(-W)$ guarantee the proper behaviour near the $W=-m_{l}$ point.

Let us introduce renormalization of fields in a standard manner

$$
\Psi=Z^{1 / 2} \Psi^{\mathrm{ren}}, \quad \bar{\Psi}=\bar{\Psi}^{\mathrm{ren}} \bar{Z}^{1 / 2} .
$$

In theories with $\gamma^{5}$ the renormalization "constants" are in fact the matrices of dimension 4

$$
Z^{1 / 2}=\alpha+\gamma^{5} \beta, \quad \bar{Z}^{1 / 2}=\bar{\alpha}+\gamma^{5} \bar{\beta} .
$$

If to consider the mixing problem of $n$ generations of fermions then $\alpha, \beta, \bar{\alpha}, \bar{\beta}$ are matrices of dimension $n$.

Inverse renormalized matrix propagator is defined by

$$
S^{\mathrm{ren}}=\bar{Z}^{1 / 2} S Z^{1 / 2}=\left(\bar{\alpha}+\gamma^{5} \bar{\beta}\right) S\left(\alpha+\gamma^{5} \beta\right) .
$$


Let us restrict ourselves by CP-conservating theory and by the case of stable fermions. CPconservation leads to the symmetry properties (3.2) and in order to keep this symmetry after renormalization we have to require ${ }^{3}$

$$
\bar{\alpha}=\alpha^{\mathrm{T}}, \quad \bar{\beta}=-\beta^{\mathrm{T}} .
$$

So, the multiplicative renormalization of inverse propagator is defined by

$$
S^{\mathrm{ren}}(p)=\left(\alpha^{\mathrm{T}}-\gamma^{5} \beta^{\mathrm{T}}\right) S(p)\left(\alpha+\gamma^{5} \beta\right) .
$$

Renormalization conditions for $\left(S^{\text {ren }}\right)_{i j}(4.2)$ can be formulated in terms of decomposition (4.3) at $\varepsilon_{l}=W-m_{l} \rightarrow 0$.

- $i=l, j=l$

$$
\begin{aligned}
& \left(S_{1}^{\mathrm{ren}}\right)_{l l} \longrightarrow W-m_{l}, \quad\left(S_{2}^{\mathrm{ren}}(W)\right)_{l l}=\left(S_{1}^{\text {ren }}(-W)\right)_{l l} \\
& \left(S_{3}^{\mathrm{ren}}\right)_{l l}=o\left(\varepsilon_{l}\right), \quad\left(S_{4}^{\mathrm{ren}}\right)_{l l}=o\left(\varepsilon_{l}\right) .
\end{aligned}
$$

- $i \neq l, j=l$

$$
\left(S_{1}^{\mathrm{ren}}\right)_{i l}=O\left(\varepsilon_{l}\right), \quad\left(S_{4}^{\mathrm{ren}}\right)_{i l}=O\left(\varepsilon_{l}\right) .
$$

Corresponding elements of $S_{2}, S_{3}$ matrices are defined by replacement $W \rightarrow-W$ and they are $O(1)$.

- $i=l, j \neq l$

$$
\left(S_{1}^{\mathrm{ren}}\right)_{l j}=O\left(\varepsilon_{l}\right), \quad\left(S_{3}^{\mathrm{ren}}\right)_{l j}=O\left(\varepsilon_{l}\right) .
$$

Elements of matrices $S_{2}, S_{4}$ are obtained by $W \rightarrow-W$.

We see that in the limit $W \rightarrow m_{l}$ there arise some conditions on $l$-th row and $l$-th column of $S_{1}$ matrix, on $l$-th row of $S_{3}$ and on $l$-th column of $S_{4}$. Matrix coefficients in decomposition (4.3) should have the following behaviour at $\varepsilon_{l}=W-m_{l} \rightarrow 0$

$$
\begin{aligned}
& S_{1}^{\mathrm{ren}} \sim\left(\begin{array}{ccccc}
O(1) & \ldots & O\left(\varepsilon_{l}\right) & \ldots & O(1) \\
\vdots & & \vdots & & \vdots \\
O\left(\varepsilon_{l}\right) & \ldots & \varepsilon_{l} & \ldots & O\left(\varepsilon_{l}\right) \\
\vdots & & \vdots & & \vdots \\
O(1) & \ldots & O\left(\varepsilon_{l}\right) & \ldots & O(1)
\end{array}\right), \quad S_{2}^{\mathrm{ren}} \sim O(1) \\
& S_{3}^{\mathrm{ren}} \sim\left(\begin{array}{c}
O(1) \\
\vdots \\
O\left(\varepsilon_{l}\right) \ldots o\left(\varepsilon_{l}\right) \ldots O\left(\varepsilon_{l}\right) \\
\vdots \\
O(1)
\end{array}\right), \quad S_{4}^{\mathrm{ren}} \sim\left(\begin{array}{c}
O\left(\varepsilon_{l}\right) \\
\vdots \\
O(1) \ldots o\left(\varepsilon_{l}\right) \ldots O(1) \\
\vdots \\
O\left(\varepsilon_{l}\right)
\end{array}\right)
\end{aligned}
$$

\footnotetext{
${ }^{3}$ It corresponds to the pseudo-Hermitian condition $\bar{Z}^{1 / 2}=\gamma^{0}\left(Z^{1 / 2}\right)^{\dagger} \gamma^{0}$ [20], but it was noted in [21] that in presence of imaginary part in self-energy this condition becomes contradictory.
} 
We use the spectral representation for inverse propagator (1.3), then the renormalized inverse propagator looks similarly

$$
S^{\mathrm{ren}}=\sum_{k=1}^{2 n} \lambda_{k}(W) \tilde{\Pi}_{k}
$$

but $\tilde{\Pi}_{k}=\left(\alpha^{\mathrm{T}}-\gamma^{5} \beta^{\mathrm{T}}\right) \Pi_{k}\left(\alpha+\gamma^{5} \beta\right)$ are not projectors in general case. Recall that projectors $\Pi_{k}$ (3.6) are expressed through some vectors $\psi_{k}, \phi_{k}$ which we suppose to be columns.

Calculating the quasi-projectors $\tilde{\Pi}_{k}$, we find that they have the same form (3.6) with renormalized vectors

$$
\tilde{\Pi}_{k}=\mathscr{P}_{1} \cdot \psi_{k}^{r}\left(\psi_{k}^{r}\right)^{\mathrm{T}}-\mathscr{P}_{2} \cdot \phi_{k}^{r}\left(\phi_{k}^{r}\right)^{\mathrm{T}}+\mathscr{P}_{3} \cdot \psi_{k}^{r}\left(\phi_{k}^{r}\right)^{\mathrm{T}}-\mathscr{P}_{4} \cdot \phi_{k}^{r}\left(\psi_{k}^{r}\right)^{\mathrm{T}}
$$

where renormalized vectors looks like

$$
\psi_{k}^{r}=\alpha^{\mathrm{T}} \psi_{k}+\beta^{\mathrm{T}} \phi_{k}, \quad \phi_{k}^{r}=\alpha^{\mathrm{T}} \phi_{k}+\beta^{\mathrm{T}} \psi_{k},
$$

Now require $S^{\text {ren }}$ in form (4.13), (4.14) to satisfy the conditions (4.12). If $W \rightarrow m_{l}$ and $\lambda_{l}\left(m_{l}\right)=0$, it is convenient to separate out the $l$-th eigenvalue in $S^{\text {ren }}$

$$
S^{\mathrm{ren}}=\lambda_{l}(W) \tilde{\Pi}_{l}+\sum_{k \neq l} \lambda_{k}(W) \tilde{\Pi}_{k}
$$

We will show that the renormalization conditions (4.12) may be formulated as requirements on the vectors $\psi_{k}(W)$. To see it, we will write the explicit form of matrices $S_{M}^{\text {ren }}(W)$, which follows from (4.13), (4.14)

$$
\begin{aligned}
& S_{1}^{\mathrm{ren}}=\sum_{k} \lambda_{k}(W) \psi_{k}^{r}\left(\psi_{k}^{r}\right)^{\mathrm{T}}=\lambda_{l}(W) \psi_{l}^{r}\left(\psi_{l}^{r}\right)^{\mathrm{T}}+\sum_{k \neq l} \lambda_{k}(W) \psi_{k}^{r}\left(\psi_{k}^{r}\right)^{\mathrm{T}}, \\
& S_{2}^{\mathrm{ren}}=-\sum_{k} \lambda_{k}(W) \phi_{k}^{r}\left(\phi_{k}^{r}\right)^{\mathrm{T}}, \\
& S_{3}^{\mathrm{ren}}=\sum_{k} \lambda_{k}(W) \psi_{k}^{r}\left(\phi_{k}^{r}\right)^{\mathrm{T}}=\lambda_{l}(W) \psi_{l}^{r}\left(\phi_{l}^{r}\right)^{\mathrm{T}}+\sum_{k \neq l} \lambda_{k}(W) \psi_{k}^{r}\left(\phi_{k}^{r}\right)^{\mathrm{T}}, \\
& S_{4}^{\mathrm{ren}}=-\sum_{k} \lambda_{k}(W) \phi_{k}^{r}\left(\psi_{k}^{r}\right)^{\mathrm{T}}=-\lambda_{l}(W) \phi_{l}^{r}\left(\psi_{l}^{r}\right)^{\mathrm{T}}-\sum_{k \neq l} \lambda_{k}(W) \phi_{k}^{r}\left(\psi_{k}^{r}\right)^{\mathrm{T}} .
\end{aligned}
$$

First of all, consider behaviour of non-diagonal elements of $S^{\text {ren }}(p)$. Looking at conditions (4.10), (4.11), one can see that they are determined by $k \neq l$ terms in sums (4.17) and are reduced to requirements on the renormalized vector $\psi_{k}^{r}(W)$, namely

$$
\left(\psi_{k}^{r}\left(m_{l}\right)\right)^{l}=0, \quad k \neq l .
$$

Renormalization of diagonal elements (4.9) is fixed by $i=l$ term in a sum and gives the condition

$$
\left(\psi_{l}^{r}(W)\right)^{l} \rightarrow c_{l} \neq 0 \quad \text { at } W \rightarrow m_{l} .
$$

Thus, the constant $c_{l}$ multiplying the eigenvalue, provides the unit slope. It is naturally to suppose it as renormalized eigenvalue

$$
\lambda_{l}^{\text {ren }}(W)=\lambda_{l}(W) c_{l}^{2} \rightarrow W-m_{l} \quad \text { at } W \rightarrow m_{l} .
$$


Thus, the spectral representation allows to reduce the renormalization of matrix propagator to much more simple problem (4.18), (4.19) of renormalization of the vector $\psi_{k}(W)$. Solution of this problem may be written in compact form without using perturbation theory. Let us show that matrices $\alpha, \beta$ can to be chosen as

$$
\alpha=\left(c_{1} \psi_{1}\left(m_{1}\right), c_{2} \psi_{2}\left(m_{2}\right), \ldots, c_{n} \psi_{n}\left(m_{n}\right)\right), \quad \beta=-\left(c_{1} \phi_{1}\left(m_{1}\right), c_{2} \phi_{2}\left(m_{2}\right), \ldots, c_{n} \phi_{n}\left(m_{n}\right)\right) .
$$

As in above, to simplify notations it's convenient to suppose the vectors $\psi_{k}(W), \phi_{k}(W)$, constructing the eigenprojectors $\Pi_{k}$, to be columns. Then the matrices (4.21) consist of columns - these vectors at fixed $W$.

Let us verify that the matrices (4.21) provide the correct renormalization properties. To this end we can calculate according to (4.15) the renormalized vector $\psi_{k}^{\text {ren }}(W)$

$$
\psi_{k}^{\mathrm{ren}}(W)=\left(\begin{array}{c}
c_{1}\left[\psi_{1}^{\mathrm{T}}\left(m_{1}\right) \psi_{k}(W)-\phi_{1}^{\mathrm{T}}\left(m_{1}\right) \phi_{k}(W)\right] \\
c_{2}\left[\psi_{2}^{\mathrm{T}}\left(m_{2}\right) \psi_{k}(W)-\phi_{2}^{\mathrm{T}}\left(m_{2}\right) \phi_{k}(W)\right] \\
\vdots \\
c_{n}\left[\psi_{n}^{\mathrm{T}}\left(m_{n}\right) \psi_{k}(W)-\phi_{n}^{\mathrm{T}}\left(m_{n}\right) \phi_{k}(W)\right]
\end{array}\right),
$$

Calculating the $l$-th component of this vector at the point $W=m_{l}$, we have

$$
\left(\psi_{k}^{\mathrm{ren}}\left(m_{l}\right)\right)^{l}=c_{l}\left[\psi_{l}^{\mathrm{T}}\left(m_{l}\right) \psi_{k}\left(m_{l}\right)-\phi_{l}^{\mathrm{T}}\left(m_{l}\right) \phi_{k}\left(m_{l}\right)\right]=c_{l} \delta_{l k},
$$

where we used the orthonormality property (3.10). So we see that vector (4.22), following from renormalization "constants" (4.21) has all necessary properties and provides the correct renormalization of inverse propagator.

\section{Conclusions}

Here we constructed the spectral representation for matrix fermion propagator in presence of P-parity violation, which gives rather compact and simple description of fermion mixing in QFT. This construction generalizes the well-known matrix spectral representation for more complicated objects with two sets of indexes.

In this representation the inverse matrix propagator has the form (1.3), where the eigenprojectors $\Pi_{i}$ are constructed (2.32) from the vectors $\psi_{i}, \tilde{\psi}_{i}$. In case of CP-conservation we get the simpler answer (3.6), which contains only one vector $\psi_{i}$ - solution of homogeneous equation (3.7). In this case in order to construct the dressed propagator, we need to solve the characteristic equation (2.19) for eigenvalues $\lambda_{i}(W)$ and to solve for every $i$ the homogeneous equation (3.7).

We investigated the multiplicative (WFR) renormalization of obtained matrix propagator. The on-shell requirements AHKKM [20] for renormalized propagator may be easily transformed into conditions for renormalized vector $\psi_{i}^{r}$ (4.18), (4.19). After it we have much more simple problem and it allows to write down the general answer for renormalization constants (4.21). Note that the answer for $Z^{1 / 2}, \bar{Z}^{1 / 2}$ looks very simple just in terms of vectors $\psi_{i}(W)$, appeared in the eigenstate problem (1.1). 
Thus, the eigenstate problem (1.1) for matrix fermion propagator may be reduced to few simple steps and it gives an elegant algebraic construction, where the poles in a propagator are separated from each other. After it the renormalization constants $Z^{1 / 2}, \bar{Z}^{1 / 2}$ may be found in a closed form without using of perturbation theory.

Certainly, there exist some open questions in framework of this algebraic construction. We mention here only the properties at substitution $W \rightarrow-W$, which connects the positive and negative energy poles, and properties of spin projectors in case of fermion mixing. These questions need a more detailed consideration.

\section{A. Completeness condition and spin projectors}

The necessary requirement in constructing of spectral representation is the completeness condition for eigenprojectors

$$
X \equiv \sum_{i=1}^{2 n} \Pi_{i}=I_{4} I_{n}
$$

Here $I_{4}$ and $I_{n}$ are unit matrices of indicated dimensions. If to represent $X$ in form of decomposition (2.3) with coefficients $X_{M}$, then (A.1) is equivalent to

$$
X_{1}=X_{2}=I_{n}, \quad X_{3}=X_{4}=0,
$$

or with use of the explicit form of projectors (3.6) (CP is conserved):

$$
\sum_{i=1}^{2 n} \psi_{i}\left(\psi_{i}\right)^{\mathrm{T}}=-\sum_{i=1}^{2 n} \phi_{i}\left(\phi_{i}\right)^{\mathrm{T}}=I_{n}, \quad \sum_{i=1}^{2 n} \psi_{i}\left(\phi_{i}\right)^{\mathrm{T}}=-\sum_{i=1}^{2 n} \phi_{i}\left(\psi_{i}\right)^{\mathrm{T}}=0 .
$$

Orthogonality of projectors $\Pi_{i} \Pi_{k}=\delta_{i k} \Pi_{k}$ leads to the property $X \cdot X=X$, i.e. $X$ may be projector or unit operator. To see that $X=1$, there are two possibilities. The first one is to show completeness (A.1) by direct calculations, similar to $n=1$ case. Another possibility is to look at the action of $X$ on an arbitrary "vector" $y_{\alpha, a}$ with two different indexes. Since an arbitrary "vector" has $4 n$ degrees of freedom, we see that $2 n$ eigenprojectors $\Pi_{i}$ give only half of the necessary. It's evident that spin degrees of freedom should be taken into account. In other words, there should exist the spin projectors $\Sigma_{i}^{ \pm}$, commutating with $\Pi_{i}$

$$
\left[\Sigma_{i}^{ \pm}, \Pi_{i}\right]=0, \quad \Sigma_{i}^{ \pm} \Sigma_{i}^{ \pm}=\Sigma_{i}^{ \pm}, \quad \Sigma_{i}^{ \pm} \Sigma_{i}^{\mp}=0, \quad \Sigma_{i}^{ \pm}+\Sigma_{i}^{\mp}=1 .
$$

In this case the eigenstate problem (both left and right) has twice as many solutions with the same orthonormality property.

$$
S\left(\Pi_{i} \Sigma_{i}^{ \pm}\right)=\lambda_{i}\left(\Pi_{i} \Sigma_{i}^{ \pm}\right) .
$$

Completeness condition takes the form

$$
\sum_{i=1}^{2 n}\left(\Pi_{i} \Sigma_{i}^{+}+\Pi_{i} \Sigma_{i}^{-}\right)=I_{4} I_{n}
$$

and inverse propagator is represented as

$$
S(p)=\sum_{i=1}^{2 n} \lambda_{i}\left(\Pi_{i} \Sigma_{i}^{+}+\Pi_{i} \Sigma_{i}^{-}\right) .
$$


Let us note that $\Sigma_{i}^{ \pm}$are some generalized off-shell spin projectors, not coinciding with the well known ones (call them as bare)

$$
\Sigma_{0}^{ \pm}=\frac{1}{2}\left(1 \pm \gamma^{5} \hat{s}\right), \quad(s p)=0, \quad s^{2}=-1 .
$$

Spin projectors become non-trivial in presence of $\mathrm{P}$-parity violation. To see it, we can consider the dressing of a single fermion $(n=1)$ in theory with $\gamma^{5}$ in a vertex. In this case the inverse dressed propagator looks as

$$
S(p)=A\left(p^{2}\right)+\hat{p} B\left(p^{2}\right)+\gamma^{5} C\left(p^{2}\right)+\hat{p} \gamma^{5} D\left(p^{2}\right)
$$

and does not commutate with the standard spin projectors $\Sigma_{0}^{ \pm}$. Nevertheless, there exist generalized spin projectors, commutating with inverse propagator. The eigenprojectors $\Pi_{i}$ for single fermion field were obtained in [16] and may be represented in the simple form

$$
\Pi_{1,2}=\frac{1}{2}\left(1 \pm \hat{n} \cdot \frac{B+\hat{n} \gamma^{5} C / W+\gamma^{5} D}{\sqrt{B^{2}+C^{2} / W^{2}-D^{2}}}\right) .
$$

The generalized spin projectors, satisfying all necessary requirements, have the following form

$$
\Sigma^{ \pm}=\frac{1}{2}\left(1 \pm \gamma^{5} \hat{s} \cdot \frac{B+\hat{n} \gamma^{5} C / W+\gamma^{5} D}{\sqrt{B^{2}+C^{2} / W^{2}-D^{2}}}\right), \quad s^{2}=-1, \quad(s p)=0 .
$$

In absence of interaction $(B=1, C=D=0)$ or in theory with parity conservation $(C=D=0)$ they coincide with the standard ones $\Sigma_{0}^{ \pm}$. So one can conclude that appearance of $\gamma^{5}$ in a vertex leads to dressing of spin projectors together with dressing of propagator.

\section{References}

[1] W. Grimus and P. Stockinger, Real oscillations of virtual neutrinos, Phys. Rev. D54 (1996) 3414.

[2] W. Grimus, P. Stockinger and S. Mohanty, The field theoretical approach to coherence in neutrino oscillations, Phys. Rev. D59 (1999) 013011.

[3] C. Giunti, Neutrino wave packets in quantum field theory, JHEP 0211 (2002) 017.

[4] M. Beuthe, Oscillations of neutrinos and mesons in quantum field theory, Phys. Rept. 375 (2003) 105.

[5] E.Kh. Akhmedov and J. Kopp, Neutrino oscillations: Quantum mechanics vs. quantum field theory, JHEP 1004 (2010) 008, Erratum-ibid. 1310 (2013) 052.

[6] D.V. Naumov and V.A. Naumov, A diagrammatic treatment of neutrino oscillations, J. Phys. G37 (2010) 105014

[7] M. Dvornikov, Field theory description of neutrino oscillations. Published in Neutrinos: Properties, Sources and Detection, ed. by J.P.Greene. (Nova Science Publishers, New York, 2011, p. 23-90. e-Print: arXiv:1011.4300

[8] J.F. Donoghue, Finite Renormalization, Flavor Mixing and Weak Decays, Phys. Rev. D19 (1979) 2772.

[9] A. Denner and T. Sack, Renormalization of the Quark Mixing Matrix, Nucl. Phys. B 347 (1990) 203. 
[10] A. Barroso, L. Brucher and R. Santos, Renormalization of the Cabibbo-Kobayashi-Maskawa matrix, Phys. Rev. D62 (2000) 096003.

[11] B.A. Kniehl and A. Sirlin, Simple On-Shell Renormalization Framework for the Cabibbo-Kobayashi-Maskawa Matrix, Phys. Rev. D74 (2006) 116003. [hep-th/0612033].

[12] B.A. Kniehl and A. Sirlin, Renormalization in general theories with inter-generation mixing, Phys. Rev. D85 (2012) 036007.

[13] B.A. Kniehl, All-Order Renormalization of the Propagator Matrix for Fermionic Systems with Flavor Mixing, Phys. Rev. Lett. 112 (2014) 071603.

[14] B.A. Kniehl, All-order renormalization of propagator matrix for unstable Dirac fermions, Phys. Rev. D89 (2014) 096005

[15] A. Messiah, Quantum mechanics, vol. 1 (North-Holland Pub. Co., 1961)

[16] A.E. Kaloshin and V.P. Lomov, Top Quark as a Resonance, Eur. Phys. J. C72 (2012) 2094.

[17] V.N. Gribov, Fermion Regge poles and the asymptotic behavior of meson - nucleon large - angle scattering, Zh. Eksp. Teor. Fiz. 43 (1962) 1529.

[18] V.N. Gribov, L.B. Okun and I.Ya. Pomeranchuk, On processes determined by fermion Regge poles, Zh. Eksp. Teor. Fiz. 45 (1963) 1114.

[19] B.A. Kniehl and A. Sirlin, Pole Mass, Width, and Propagators of Unstable Fermions, Phys. Rev. D77 (2008) 116012.

[20] K.I. Aoki, Z. Hioki, M. Konuma, R. Kawabe and T. Muta, Electroweak Theory. Framework of On-Shell Renormalization and Study of Higher Order Effects, Prog. Theor. Phys. Suppl. 73 (1982) 1.

[21] D. Espriu, J. Manzano and P. Talavera, Flavor mixing, gauge invariance and wave function renormalization, Phys. Rev. D66 (2002) 076002. 